\title{
Historias fuera de rango: escuchas tramadas en la era de las extinciones (sobre Jana Winderen)
}

\author{
Susana Jiménez Carmona \\ Universidad de Barcelona \\ susanajimenezcarmona@gmail.com
}

RESUMEN: Este artículo se acerca a la práctica y el trabajo de Jana Winderen para reflexionar sobre el arte que implica a seres vivos no humanos como protagonistas. Esta artista ha centrado su trabajo principalmente en animales a los que somos sordos, sintiendo una predilección especial por las pequeñas criaturas marinas cuyas vidas se ven profundamente afectadas por la acción de los humanos. En diálogo con Ursula Le Guin, Donna Haraway, Thom van Dooren, Vinciane Despret y Rosi Braidotti, nos preguntamos si es posible acercarnos a estos otros seres vivos y sus mundos de maneras no predominantemente antropocéntricas y qué papel pueden desempeñar las prácticas artísticas tentando resistencias y posibilidades que hagan este mundo habitable para humanos y no humanos.

PALABRAS CLAVE: Jana Winderen; Arte sonoro; Disposición; Etología; Multiespecie; Historias; Interdependencia.

\section{Stories out of Range: Listenings Woven in the Era of Extinctions (about Jana Winderen)}

ABSTRACT: This article suggests delving into the practice and work of Jana Winderen in order to reflect on the art involving non-human living beings as protagonists. This artist has focused her work mainly on living beings to whom we are deaf, feeling a special predilection for the tiny marine creatures whose lives are deeply affected by the action of humans. In dialogue with Ursula Le Guin, Donna Haraway, Thom van Dooren, Vinciane Despret and Rosi Braidotti, we ask if it is possible to approach these other living beings and their worlds in ways that are not predominantly anthropocentric and if artistic practices can play a role in the trying resistances and feeling possibilities which make this world inhabitable for humans and non-humans.

KEYWORDS: Jana Winderen; Sound art; Disposition; Ethology; Multispecies; Stories; Interdependence.

Recibido: 27 de febrero de 2019 / Aceptado: 21 de junio de 2019.

Cada sujeto teje relaciones, como hilos de una araña, sobre determinadas propiedades de las cosas, entrelazándolas hasta configurar una sólida red que será portadora de su existencia.

Uexküll, 2016: 52

Living well with others can never be about just learning to tell new stories; it must also involve learning new kinds of attentiveness to the stories of others

- even if they are unspoken or are told in other-than-human languages».

Dooren, 2016: 78

\section{Hormigas y camarones}

En The Compass Rose, una compilación de textos que Ursula Le Guin publicó entre 1974 y 1982, se encuentra «El autor de las semillas de acacia» y otros extractos del Diario de la Sociedad de Therolingüistas. Este hermoso y breve texto nos va a servir para introducir e hilar este artículo que, aunque está centrado en el trabajo de la artista Jana Winderen, se tra-

Cómo citar este artículo: JIMÉNEZ CARMONA, Susana, «Historias fuera de rango: escuchas tramadas en la era de las extinciones (sobre Jana Winderen)", Boletín de Arte-UMA, n. ${ }^{\circ}$ 40, Departamento de Historia del Arte, Universidad de Málaga, 2019, pp. 159-166, ISSN: 0211-8483, e-ISSN: 2695-415X, DOI: http://dx.doi.org/10.24310/ BoLArte.2019.v0i40.5669 
ma con otras voces humanas y no humanas. El texto de Le Guin empieza así:

\begin{abstract}
Manuscrito encontrado en un hormiguero
Los mensajes, escritos con la exudación de glándulas táctiles, fueron hallados sobre la superficie de semillas de acacia no germinadas colocadas en hilera al final de un túnel estrecho e irregular, posiblemente una desviación de otro túnel más profundo de la colonia. Lo primero que llamó la atención de los investigadores fue la posición ordenada de las semillas.
\end{abstract}

Los mensajes son fragmentarios y la traducción aproximativa y altamente interpretativa; pero el texto parece digno de interés, aunque solo sea por su sorprendente falta de parecido con cualquier otro texto fórmico conocido.

Semillas 1-13

No tocar(é) las antenas. No golpear(é). Verter(é) sobre secas semillas (mi) dulzura de alma. Se pueden encontrar cuando (yo haya) muerto. ¡Palpa esta seca madera! ¡Avis(o)! i(Yo estoy) aquí!

Como alternativa, este pasaje puede leerse:

No toqu(es) las antenas. No golpe(es). Viert(e) sobre secas semillas (tu) dulzura de alma. (Otros) pueden encontrarlas cuando (hayas) muerto. ¡Palpa esta seca madera! Avisa: ¡(Estoy) aquí!

En el no muy conocido dialecto de las Hormigas no se emplea ningún pronombre personal, excepto los de la tercera persona de singular y plural, y la primera del plural. En este texto solo aparecen las raíces de los verbos; por lo que no podemos decidir si se trata de una autobiografía o un manifiesto 1.

Mientras realizaba unas grabaciones subacuáticas en la costa noruega, a Winderen le llamaron la atención unos sonidos crujientes y constantes cuya exacta autoría desconocía. Esta artista, que además de bellas artes ha estudiado matemáticas, química y ecología marina, comenzó a investigar y a preguntar a diferentes especialistas sobre el género de camarón del que provendrían estos sonidos. Winderen comenta que:

Las respuestas que me llegaron sugieren que los sonidos son probablemente producidos cuando los camarones se alimentan. Ya sé que los camarones hacen sonidos cuando gol- pean, cuando raspan para paralizar a sus presas, pero ¿los otros miembros de la misma familia hacen lo mismo? Nadie parece saberlo. Bajo el agua, conocemos muy poco los paisajes sonoros creados por las criaturas vivas y comprendemos poco los detalles de las variaciones entre los diferentes gruñidos, ruidos de choque y rumores producidos por el bacalao, el eglefino o el abadejo, y cómo viven y se orientan mediante el uso del sonido².

En 2009, Winderen publicó The Noisiest Guys on the Planet, obra para casete dedicada enteramente a estos pequeños y ruidosos habitantes de los mares ${ }^{3}$. El trabajo de esta artista es conocido principalmente por sus grabaciones sonoras de animales marinos, si bien comenzó más interesada por los insectos, entre ellos las hormigas, a las que seguía con sus micros hasta los profundos túneles de sus hormigueros, como los therolingüistas de Le Guin (Neset, 2012: 90)4. Estas grabaciones las muestra en instalaciones y también en conciertos que, debido al número y la situación de los altavoces, la disposición del público y su elaboración site specific, se aproximan más a lo que sería una instalación que a un concierto al uso. Este interés por quienes viven bajo las aguas hace que su trabajo esté profundamente marcado por las extinciones masivas que estamos viviendo en la actualidad. Este artículo quiere poner en diálogo la obra de Winderen con pensadoras y pensadores que reflexionan acerca las relaciones entre humanos y no humanos situándose en la complejidad y gravedad del momento presente como son Vinciane Despret, Rosi Braidotti, Donna Haraway o Thom van Dooren. Tejiendo diferentes pensamientos, sonidos y narraciones queremos reflexionar sobre si el arte que escucha a seres vivos no humanos puede ayudarnos no solo a escapar de la parálisis que nos amenaza y atenaza en estos tiempos de catástrofe, sino también a abrir posibles que hagan de este un mundo más habitable para los diferentes seres que lo pueblan.

\section{Cifras y voces}

Winderen forma parte del elenco cada vez mayor de artistas sonoros interesados por la cuestión ecológica, entre quienes se encuentran, por ejemplo, Hildergard Westerkamp, Chris Watson, Leah Barclay, Christopher DeLaurenti o Xime- 
na Alarcón. Sin duda, la preocupación por nuestro entorno y la supervivencia de los demás habitantes de nuestro planeta está cada vez más extendida entre los artistas, así como en el resto de la población. No en vano, cada día podemos leer o escuchar sobre nuevas noticias o investigaciones acerca del cambio climático, la contaminación, la deforestación, etc., y sus consecuencias. Sin embargo, las cifras son tan contundentes e implacables que no es de extrañar que la sensación de impotencia sea una respuesta extendida.

El filósofo medioambiental y antropólogo Thom van Dooren se acerca a esta reacción en algunos de sus textos sobre aves buscando alternativas que permitan pensar y hacer permaneciendo en el problema (staying with the trouble), en palabras de Donna Haraway que Dooren toma. Él propone que, sin olvidar esos terribles números, se atienda tanto a la singularidad de cada forma de vida, aplicando esta noción wittgensteineana también a las comunidades de seres vivos no humanos (Dooren, 2016: 7-8), como al dolor particular de cada individuo (Dooren, 2010: 272-273). Está en juego mucho más que la diversidad.

Empecemos por el segundo foco. En principio, el arte sonoro y sus tecnologías centradas en la escucha parecen propicios para poner en práctica este acercamiento atento a cada individuo sugerido por Dooren. Sin embargo, no es tan fácil. Muestra de ello son los numerosos archivos sonoros y algunas prácticas artísticas que tienden a abstraer, y con ello a objetualizar, los sonidos de los seres vivos no humanos. Sin duda, los archivos sonoros buscan principalmente conservar y clasificar los sonidos que producen las diferentes especies de animales. Sin embargo, al hacerlo en tanto que especies y al aislarlos de las formas de vida de la que son parte no pueden evitar abstraerlos al convertir el canto de cada individuo en el canto de un «ejemplar». Otro caso es el de las prácticas acusmáticas que, en principio, parecen muy próximas al hacer de Winderen ya que también requieren de grabaciones de campo, instalaciones sonoras y conciertos semejantes a una instalación. No obstante, al perseguir la escucha reducida propuesta por Pierre Schaeffer, es decir, borrar el origen de aquello que suena, los sonidos capturados se convierten en objetos sonoros sin cuerpos ni circunstancias (2003: 56-59; 159-169). Para Winderen, esta manera de acercarse a esos otros mundos sonoros supone una falta de respeto hacia seres vivos sintientes e inteligentes (Doktor, 2014). Se trata de inteligencias otras que se comunican entre sí y con su entorno mediante sonidos (Fischer, 2010). Por ello, esta artista solo edita sus «voces»-expresión que ella utiliza- lo necesario para hacerlas más claras a nuestra escucha, por ejemplo, ralentizando las frecuencias que están fuera de nuestro rango de audición. Aunque esta ralentización de frecuencias que nos permite escuchar voces a las que somos sordos es utilizada por Winderen en gran parte de su trabajo, es protagonista principal de Out of range (2014), construida con silbidos, ritmos, crujidos o «cantos» de murciélagos, delfines, ballenas, polillas y otros animales ${ }^{5}$. Los ultrasonidos y ecolocalizaciones que traman esta obra fueron recogidos en Central Park y East River en Nueva York, un bosque en las afueras de Kaliningrado, Regents Park en Londres y varios lugares en Madeira, Noruega, Dinamarca y Suecia.

\section{Disposiciones y cantos}

Antes de seguir, volvamos un momento a Dooren y a su propuesta de atender al dolor de cada ser singular, pues cabría preguntarse si esta disposición no podría resultar asimismo paralizante. Es lo que le sucede, por ejemplo, al protagonista de The sound machine de Roald Dahl, que enloquece al ser capaz de escuchar, gracias a una máquina ingeniada por él, la voz y los lamentos de cada planta al ser cortada (Dahl, 2011). Para tratar de responder a esa cuestión, retomemos brevemente «El autor de los granos de acacia» de Le Guin. Pocos párrafos después del Manuscrito encontrado en un hormiguero, podemos leer cómo el doctor Petri reclama la necesidad de abandonar los prejuicios que guían la literatura cinética para ser capaces de apreciar a animales como el pingüino emperador que, «casi inmóvil, inefable y sutil», encierra su poesía en «el roce de una pluma, el movimiento de un ala, el contacto ligero y cálido de quien está al lado» (Le Guin, 2015: 11s). El propio presidente de la sociedad de los Therolingüistas afirma contundente unas líneas después que apenas hemos extendido nuestra mirada más allá de nosotros mismos. Apenas hemos tendido nuestros oídos a nuestro alrededor, diría Winderen.

Esta artista recorre tierras y mares cargando con un pesado equipo de grabación que le permite oír sonidos que están fuera del rango de audición humana, sonidos producidos por seres que, aunque nos parezcan lejanos, se 
encuentran, eso sí, bajo nuestro rango de acción ${ }^{6}$. Sin embargo, con la tecnología sola no se alcanza esta escucha atenta, como hemos señalado arriba ${ }^{7}$. Es necesario ponerse en sus pieles y sus oídos -u otros órganos perceptores del sonido-, así como situarse en sus hábitats ${ }^{8}$. Este situarse no solo requiere del trabajo previo de investigación, sino que exige la experiencia corporal del propio lugar en el que se graba, incluso en casos tan extremos como el Ártico, en el que frecuentemente trabaja Winderen y que pueden recordarnos a lo descrito por el doctor Petri en el texto de Le Guin. Pero, sobre todo, exige preguntarse qué pueden hacer y cómo pueden sonar esos seres vivos (como en el caso de los camarones). Por tanto, es necesario preguntarse por sus modos de existencia para poder así acercarse a sus singulares formas de vida. Nos encontramos, de este modo, con una puesta en práctica de la etología dentro del ámbito del arte sonoro. Esta ciencia de lo que los seres hacen y hacen hacer que Deleuze plantea en sus cursos sobre Spinoza es tomada, posteriormente, por Vinciane Despret en sus textos sobre comunicaciones entre humanos y no humanos ${ }^{9}$ -y por Dooren en sus estudios sobre las formas de vida de las aves-.

Despret dedica parte importante de su trabajo al estudio de los modos de afectarse mutuamente que permiten que humanos y animales nos comuniquemos de maneras no verbales. Para que esta mutua afectación se produzca, esta pensadora considera necesario que se den sensibilidades y percepciones que propicien una disponibilidad hacia los otros que escape de o, al menos, cuestione la creencia en la excepcionalidad humana frente al resto de seres vivos. Despret apuesta por buscar otras maneras de expresarse, de moverse, de sentir que sean ajenas a los prejuicios y esquemas tanto del logocentrismo como de esa visión a distancia que todo lo objetualiza y permite al sujeto (humano) sentirse al margen y a salvo. La disponibilidad de la que habla Despret es una disponibilidad entre cuerpos que pueden ser afectados: "Con la noción de "disponibilidad" los signos que caracterizan al mundo y al sujeto se redistribuyen de forma distinta. Ambos, sujeto y mundo, están activos y se transforman en función de la disponibilidad del otro. Ambos se articulan mediante lo que el otro "les hace hacer"» (2008: $250)^{10}$.

En Winderen encontramos esta disponibilidad hacia los otros, a la vez que se reconoce como una oyente hu- milde consciente de sus propias limitaciones sensoriales y corporales, aunque se vean parcialmente salvadas por las tecnologías que emplea, en un devenir máquina que recuerda al posthumano de Braidotti ${ }^{11}$. En su hacer audibles aquellos seres a los que somos sordos, no solo va aprendiendo acerca de sus modos de existencia, sino también a ser afectada por ellos. Podríamos hablar aquí de una escucha que cuestiona la excepcionalidad humana y disloca nuestro antropocentrismo al ponerse en disposición de, al estar atenta a lo que otras voces, otros cuerpos no humanos suenan y cuentan. Estas otras voces y otros cuerpos pueden en ocasiones ser vecinos tan próximos a los humanos, y tan poco apreciados por estos, como las ratas de ciudad, a las que Winderen dedica una instalación en 2017: Rats - Secret Soundscapes of the City. Siguiendo sus cantos de amor, entonados en gran parte mediante ultrasonidos, la artista nos introduce en sus particulares paisajes sonoros, unos paisajes que compartimos con ellas aunque no seamos capaces de oírlos. Esta sordera es paralela a la profunda huella sonora que los humanos dejamos en estos soundscapes: a lo largo del día y de la noche provocamos sonidos tremendamente fuertes que molestan y dañan a los otros habitantes de nuestras ciudades multiespecies. En sus instalaciones y conciertos, esta artista busca que el público tienda también su piel y sus oídos para dejarse afectar por estos seres y por estos sonidos vivos con los que estamos inevitablemente conectados. No trata de convencer tal como podría hacerlo con un discurso, ni de darnos pautas o soluciones concretas, sino de afectar cómo nos relacionamos con unos seres complejos y sensibles que muchas veces solo conocemos servidos en un plato $^{12}$, como suele suceder con el bacalao, animal al que dedica su trabajo Heated (Fischer, 2010)13. Winderen desea que, gracias a sus obras, las personas se paren a escuchar con atención, quieran saber más y promuevan el respeto hacia los demás seres vivos y sus formas de vida (Doktor, 2014). Se trataría, pues, de una práctica artística que busca convertirse en una práctica del cuidar pasando por el conocer, parafraseando a Despret (2008: 257).

\section{Redes e historias}

Saber más sobre los otros, sobre sus formas de vida, no solo nos permite percibir de manera diferente lo que nos 
rodea, sino también urdir otro tipo de relaciones, porque de este «lo que nos rodea» no somos ajenos, ni excepción sino parte profundamente tramada. Esta inmanente ontología relacional sostiene el trabajo de Winderen. $Y$ desde ella reflexionan Braidotti, Dooren y Haraway sobre el actual momento antropocénico. Precisamente, para estos tres pensadores, este momento crítico que estamos viviendo nos coloca en un momento único para darnos cuenta de las relaciones de interdependencia que hacen posible la vida en este planeta, algo por lo que cada vez más artistas se sienten interpelados.

Hemos visto arriba cómo Dooren proponía atender a las singularidades de cada forma de vida que está en juego, así como a cada individuo que sufre y a su dolor particular, para que puedan surgir otros modos de pensar y hacer. $Y$ también nos preguntábamos si este acercamiento no podría ser asimismo paralizante. La respuesta está en cómo se entiende esta individualidad. El individuo al que apunta Dooren no es el sujeto aislado, sino que se trataría en cada caso de una particular red de relaciones (2010: 272; 2017: 12). Ahora bien, esta relacionalidad poco tiene que ver con el holismo que propugna que todo está relacionado con todo y que tan poco invita a actuar. Dooren defiende que «si bien es posible que, al final, todos estemos conectados unos con otros, la especificidad y la proximidad de las conexiones son importantes» a la hora de propiciar la acción. Por ello considera más adecuado dibujar la trama desde un «todo está conectado a algo que a su vez está conectado a otra cosa» (Dooren, 2016: 60) ${ }^{14}$. Posicionarse en este tipo de relacionalidad situada es una apuesta por propiciar modos de entender(se), afectar(se) y hacer(se) que quizá hagan de este mundo un lugar más habitable para humanos y no humanos. $Y$ aunque no haya certeza alguna, es importante encontrar modos de vincularse que no solo respondan de manera reactiva, desde la vulnerabilidad de los peligros compartidos, sino que apunten a la dimensión positiva del flujo de relaciones con los múltiples otros, como insiste Braidotti (2013: $65,99)$. Para esta pensadora profundamente spinozista, lo que necesitamos en este momento es la alegría de una ética afirmativa que nos permita abrirnos a redefinir las interconexiones trans-especies desde un zoe-igualitarismo que abandone el excepcionalismo humano sin obviar ni la responsabilidad humana en la grave situación actual, ni nuestra capacidad de dar respuestas plurales, diversas y, por qué no, adecuadas, aunque no sean coherentes (Bignal y Braidotti, 2018: 4; Braidotti, 2018).

Entre esas respuestas plurales, la narración, la imaginación y el arte juegan un importante papel para Haraway. Esta pensadora insiste en numerosas ocasiones en la importancia de conocer y señalar las innumerables relaciones simbióticas, en su mayor parte desconocidas, de las que dependemos los seres vivos de este planeta. Precisamente, la narración, la imaginación, el arte, son claves en el señalar y sentir que, irremediablemente, vivimos-con y morimos-con otros seres vivos, así como para explorar mundos posibles que siempre serán un devenir-con esos otros (Haraway, 2016: 28, 34, 40). Estos entrelazados «con» implican una responsa-habilidad que permitiría salir del temor que nos paraliza, al permanecer en el problema tentando otras escuchas, otras afectaciones, otras subjetividades simbióticas, otros saberes trazados junto con otros seres singulares, perceptores, sintientes e inteligentes. Y ello sin pretensiones, mundanamente, con historias y prácticas ligadas a la tierra o el mar. Para Haraway, Le Guin es un ejemplo claro de esta práctica imaginativa que busca otros posibles y otros mundos más habitables, no solo para los humanos, narrando historias.

El trabajo de Winderen sería igualmente un ejemplo de práctica artística que logra hacer emerger relaciones simbióticas desconocidas, complejas, frágiles, gracias a su disposición hacia los otros, por su escucha atenta de aquellos a los que no oímos, pero con los que vivimos, morimos, devenimos y sonamos. Esta relacionalidad emerge en la obra de esta artista al construir historias, como ella misma afirma, sobre los lugares en los que graba y las criaturas que los habitan (Doktor, 2014; Wullen, 2010). Estas historias se traman con las atmósferas de los lugares que habitan y construyen humanos y no humanos y con las historias que cuentan, de singulares y diversas maneras, tanto las gentes como los demás seres vivos en su sonar, en su afectarse y afectar, en su hacer y hacerse, en su vivir-con y en su morir-con ${ }^{15}$. Winderen nos hace audibles de manera vinculante los hábitats y sus habitantes, que en su sonar se relacionan entre sí y con otros, incluyendo a los humanos (Neset, 2012).

Entre los diferentes trabajos de esta artista, queremos destacar un proyecto por el modo en el que muestra la complejidad del vivir-con y morir-con: Silencing of the reefs ${ }^{16}$. Realizado entre 2011 y 2014, este proyecto explora una de 
las zonas más frágiles y ricas en diversidad de formas de vida de nuestro planeta: los arrecifes de coral. Winderen recorre diferentes arrecifes para escuchar su «salud» mediante el sonar de sus diversos habitantes. Belize, Honduras, Brasil, Chile, isla de Cocos, Costa Rica, Panamá, islas Galápagos, la costa californiana o el mar de Cortez son algunas de las localizaciones de este proyecto. En colaboración con comunidades locales y con científicos locales e internacionales, graba desde los sonidos de los más diminutos o más amenazados habitantes de estos ecosistemas hasta el ruido de los barcos que pescan ilegalmente a los peces loro (en peligro de extinción) o cómo el estado de los arrecifes afecta asimismo a los manglares. Todas estas horas de delicada escucha y conversación se han materializado tanto en instalaciones sonoras como publicaciones de divulgación científica y programas de radio junto a miembros de Reef Check Dominican Republic.

Pero estas historias, no solo explican «las relaciones existentes, las historias también pueden conectarnos con los otros de nuevas maneras» (Dooren, 2016: 10). Winderen intenta no quedarse en la denuncia impotente o en la mera recolección de sonidos extraños, originales o exóticos condenados a desaparecer. Haciendo sonar las frágiles redes de las que dependemos los seres vivos, busca provocar una alteración que permita modificar nuestra relación con esos otros y con nuestro entorno, un entorno expandido ya que nuestras acciones atraviesan los mares más lejanos y afectan a criaturas que apenas conocemos pero cuyo devenir también nos afecta de maneras insospechadas. Posiblemente sea en prácticas colaborativas como la que desarrolla en Silencing of the reefs en las que esta intención muestre mayor contundencia, ya que es más fácil quedarse solo con el sonido de una manera reificada cuando nos sumergimos en una instalación sonora o se escucha un disco. Sin embargo, no se ha de menospreciar la capacidad de afectación de estos medios, la propia Winderen se ha encontrado con oyentes profundamente afectados al descubrir las voces de esos seres complejos y sensibles a los que esta artista dedica sus instalaciones o discos como Heated (Fischer, 2010). No obstante, la especial fuerza de Silencing of the reefs proviene de ser en su desarrollo una puesta en práctica de ese pensar-con y ese hacer-con que propicia un devenir-con: a la vez que se descubren y emergen relaciones inauditas, se van tramando ya otros lazos entre cuerpos, escuchas, lugares, saberes, memorias, deseos, afectos, vidas y muertes de humanos y no humanos. Otros lazos que no tienen por qué ser mejores. Se trata de tentar sin certezas y situados en el problema.

Sin duda, el trabajo de Jana Winderen es un bello ejemplo del modo en el que algunos artistas se están preguntando cómo habitar este mundo en tanto que artistas hoy (Pardo, 2016). Tendiendo el oído alrededor y prestando atención a la multiplicidad de formas de vidas que pueblan este planeta, cada vez son más quienes buscan trazar resistencias y posibilidades que permitan aspirar a futuros sostenibles y a construir presentes vivibles para humanos y no humanos (Braidotti, 2013: 228). 


\section{Notas}

1 Traducción propia de Le Guin, 2015: 8-9.

2 Traducción propia (<http://www.janawinderen.com/news/the_noisiest_guys_on_the_plane.html)>.

$3<$ https://soundcloud.com/janawind/the-noisiest-guys-on-the, https://soundcloud.com/janawind/the-noisiest-guys-on-the-1>.

4 Sobre la comunicación química o sonora de las hormigas puede consultarse en Hölldobler y Wilson, 2009: 167-312.

$5<$ https://janawinderen.bandcamp.com/album/out-of-range>.

6 «In form both visible and invisible to the naked eye, the waste of human societies circulates in vast atmospheric systems and oceanic currents» (Dooren, 2016: 32).

7 Es necesario apuntar que los aparatos de captación y grabación de sonido no escapan de la cadena contaminante que termina afectado a todos los seres que habitan este planeta. Winderen es consciente de ello. En lugar de rechazar esta tecnología, intenta que el impacto ecológico de las mismas sea el menor posible construyendo ella sus propias herramientas de registro sonoro y reciclando los materiales en la mayor medida de sus posibilidades.

8 Winderen se sitúa en estos hábitats no solo estudiándolos o escuchando durante las grabaciones, sino que se relaciona de manera activa con las poblaciones locales: «Of course I read about the environment I am going to, and once I am there, I speak with local people and scientists about local issues. I sometimes suggest hypotheses, and ask the biologists if they make sense; even if they would be impossible to prove, I think it is interesting to suggest them to make both myself and the audience think about what is at stake» (Neset, 2012: 93).

9 Despret hace explícito este vínculo con Deleuze: «The science of ethology is above all a practical science. It is the science of what beings do and get others to do, what they are capable of doing. Therefore, the facts that it describes should only be described using the infinitive. This definition of ethology is actually the one proposed by Gilles Deleuze, in his lectures on Baruch Spinoza's Ethics (1677)» (Despret, 2016).

10 Dooren critica a Despret por su manera de plantear las relaciones de afectación mutua entre humanos y aves cuando se pone en juego la impronta (como es el caso de los ejemplos que toma de Lorenz), pues implican para él un grave problema ético (Dooren, 2016: 102).

11 «El eje del devenir máquina resquebraja la distinción entre humanos y circuitos tecnológicos, introduciendo relaciones mediadas tecnológicamente y entendiéndolas como fundamentales para la constitución del sujeto" (Braidotti, 2013: 84).

12 La relación con los animales en tanto que comida o fuerza de trabajo, es uno de los tres niveles de consumo que mantenemos con ellos que son señalados por Braidotti por estarse redefiniendo actualmente. Los otros dos son los animales en tanto soporte emocional (animales de compañía) y como valores de metaforización y entretenimiento (Braidotti, 2018).

$13<$ https://janawinderen.bandcamp.com/album/heateds.

14 La traducción es propia.

15 La pregunta por quiénes son parte activa en estar contar que plantean Dooren y Rose (2012) es del todo pertinente aquí.

$16<$ <ttp://www.janawinderen.com/fieldtrips/sielcing_of_the_reefs_silverba.htm|\#.UUaUHI7HSYc>; <http://www.janawinderen.com/fieldtrips/silencing_of_the_ reefs_panama.html https://soundcloud.com/janawinds.

\section{Bibliografía}

BIGNAL, Simone and BRAIDOTTI, Rosi (2018), «Posthuman Systems», en Posthuman Ecologies. Complexity and Process after Deleuze, Rowman \& Littlefield, London.

BRAIDOTTI, Rosi (2013), Lo Posthumano, Editorial Gedisa, Barcelona.

- (2018), «Volverse animal». En: <https://www.cccb.org/es/multimedia/videos/volverse-animal/230241\#> (fecha de consulta: 09-07-2019).

DAHL, Roald (2011), «The Sound Machine», en Someone Like You, Penguin Books, London.

DESPRET, Vinciane (2008), «El cuerpo de nuestros desvelos: Figuras de la antropo-zoogénesis», en SÁNCHEZ-CRIADO, Tomás (ed.), Tecnogénesis. La construcción técnica de las ecologías humanas, AlBR, Madrid, pp. 229-261.

- (2016), «Bring the Dead into Ethology», en LATOUR, Bruno y LECLERCQ, Christophe (eds.), Reset Modernity!, MIT Press, pp. $450-454$.

DOKTOR, Thomas (2014), «Aufnahmen des Unhörbaren Die norwegische Künstlerin Jana Winderen sammelt besondere Geräusche», en Deutschlandfunk Kultur. En: <https://www.deutschlandfunkkultur.de/klangkunst-aufnahmen-des-unhoerbaren.1153.de.html?dram:article_id=274732> (fecha de consulta: 17-02-2019)

DOOREN, Thom van (2010), «Pain of Extinction. The Death of a Vulture», Cultural Studies Review, vol. 16, n. ${ }^{\circ} 2$, pp. $271-89$.

- (2016), Flight Ways. Life and Loss at the Edge of Extinction, Columbia University Press, New York.

- (2017), «Spectral Crows in Hawai'i: Conservation and the Work of Inheritance», en ROSE, Deborah, DOOREN, Thom van and CHRU-

LEW Matthew (eds.), Extinction Studies: Stories of Time, Death and Generations, Columbia University Press, New York. pp. $187-215$. DOOREN, Thom van and ROSE, Deborah Bird Rose (2012), «Storied-placed in a multispecies city», HUMaNIMALIA 3:2, pp. 1-27. 
FISCHER, Tobias (2010), «Interview with Jana Winderen», en Tokafi. En: <http://www.tokafi.com/15questions/interview-jana-winderen/> (fecha de consulta: 15-02-2019).

HARAWAY, Donna J. (2016), Staying with the Trouble. Making Kin in the Chtulucene, Duke University Press, Durham and London.

HÖLLDOBLER, Bert and WILSON, Edward O. (2009), The Super-Organism. The Beauty, Elegance, and Strangeness of Insect Sociaties, W.W. Norton \& Company, New York.

LE GUIN, Ursula (2015), «The Author of the Acacia Seeds. And Other Extracts from the Journal of the Association of Therolinguistics», en The Compass Rose, Gateway, London.

NESET, Anne Hilde (2012), «Monde Aquatique / Under Waters», ARTnord, n. ${ }^{0}$ 11, pp. 90-93.

PARDO SALGADO, Carmen (2016), «Une musique pour habiter le monde», en SOLOMOS, Makis, BARBANTI, Roberto, LOIZILLON, Guillaume, PAPARRIGOPOULOS, Kostas y PARDO, Carmen (eds.), Musique et écologies du son. Propositions théoriques pour une écoute du monde, L'Harmattan, Paris, pp. 197-207.

PEZANOSKI-BROWNE, Alison (2015), «The Tragic Art of Eco-Sound», The Politics of Sound Art, Leonardo Music Journal, vol. 25, pp. 9-13. SCHAEFFER, Pierre (2003), Tratado de los objetos musicales, Alianza Editorial, Madrid.

UEXKÜLL, Jakob von (2016), Andanzas por los mundos circundantes de los animales y los hombres, Editorial Cactus, Buenos Aires.

VILLETTE, Agnès (2016), «Echoes from a Fragile Ecology. Agnès Villette in conversation with Jana Winderen». DUST issue \#10 METANOIA. En: <http://www.agnesvillette.com/wp-content/uploads/2017/05/Echoes_-ilovepdf-compressed.pdf> (fecha de consulta: 9-11-2018). WITTGENSTEIN, Ludwig (2003), Investigaciones filosóficas, Instituto de Investigaciones Filosóficas UNAM, México D.F.

WULLEN, Peter (2010), «Muziek en ecologie - Interview met Jana Winderen». Cutting Edge. En: <http://www.cuttingedge.nl/interviews/ muziek-en-ecologie-interview-met-jana-winderen> (fecha de consulta: 9-11-2018). 